\title{
Research on Reliability of Straight Thread Connection of Reinforcement Cage of Bridge Foundation
}

\author{
Jinhui $\mathrm{Li}^{1}$, Xiaodong Shang ${ }^{2 *}$ and Minmin Yuan ${ }^{2}$ \\ ${ }^{1}$ Huang Mao Hai Link Management Center, Zhuhai, Guangdong Province, 519055, china \\ ${ }^{2}$ Research Institute of Highway, Ministry of Transport, Beijing 100088, China
}

\begin{abstract}
With the continuous improvement of bridge construction technology in our country, although the service life of the bridge has been greatly extended, the mechanical connection technology of steel bar in the technology is still in the stage of steel bar straight thread connection, which is nearly 30 years behind that of foreign countries. According to the development status and application requirements of bridge construction in China, it is of great practical significance to explore new technologies and methods to solve the problem of insufficient strength of straight thread connection of reinforcement. In this paper, the reliability of the straight thread reinforcement connection in the bridge foundation reinforcement cage connection technology is studied as the main breakthrough point, and the main problems encountered in the application of the straight thread reinforcement connection technology in the bridge reinforcement cage connection are analyzed.
\end{abstract}

\section{Introduction}

Bridge construction in China's road traffic construction is an indispensable and important part, but also in the road traffic construction in the core of the project. With the continuous improvement of bridge construction technology in China, the design service life of Bridges is greatly extended, up to 120 years. However, the steel reinforcement mechanical connection technology in bridge construction is still stuck in the straight thread connection of steel reinforcement 30 years ago. The appearance of the cone-sleeve joint locking technology matching with the reinforcing steel parts connected with the world-class bridge construction technology greatly improves the quality and reliability of reinforcing steel mechanical connections. This paper focuses on the advantages and disadvantages of straight thread connection technology in bridge engineering.In the future, the development direction of reinforcement connection technology in bridge engineering is to develop taper sleeve joint locking reinforcement connection technology and improve steel structure.

\section{Steel bar straight thread connection technology characteristics}

Since the 1980s, steel bar mechanical connection technology has been widely used in bridge construction, such as the sleeve extrusion connection of Humen Bridge (1993), upsetting straight thread steel bar connection of Tianjin Haihe Bridge (1998). Compared with the traditional lap joint and welding joint, it has certain advantages in terms of quality and construction efficiency, especially in the way of reinforcing steel bar straight thread connection. Since The beginning of Tianjin Haihe Bridge, the straight thread reinforced bar connection technology has been widely used in bridge engineering in China. There are three kinds of steel bar straight thread connection according to its processing technology: upsetting straight thread, direct rolling straight thread, and ribbed rolling straight thread. The joint pictures of the three processes are shown in Figure 1.
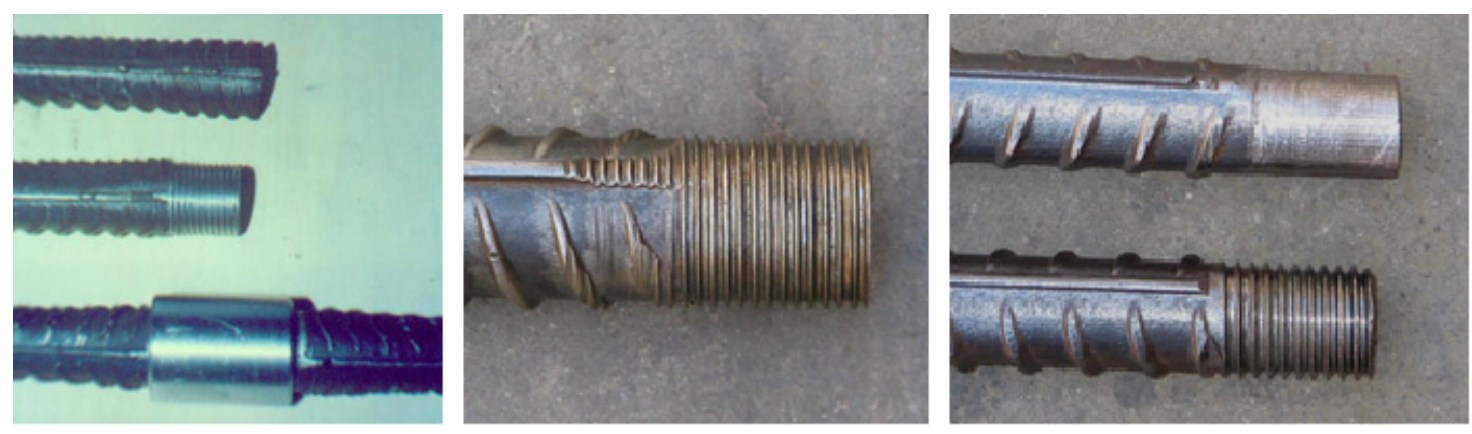

Fig 1. Three Kinds of Straight Thread Joint

\footnotetext{
*Corresponding author's e-mail: xd.shang@rioh.cn
} 


\subsection{Upsetting straight thread connection}

The end of the steel bar is upsetting first, and then the thread is machined on the outside surface of the steel bar, which has no damage to the sectional area of the steel bar, and the connection quality is better. However, the processing needs upsetting, turning thread two processes, processing equipment, more personnel, high processing cost, construction units do not want to use. Sometimes to the steel bar of individual steel mill, upsetting process is not suitable and easy to produce unqualified connection. In order to avoid this situation, less upsetting is often used on site, so it is easy to cause the appearance of the thread shape is not full, quality inspection on the appearance of the steel thread is often easy to have complaints.

\subsection{Direct rolling straight thread connection}

Straight thread is formed and rolled out on the surface of the steel bar by the steel bar wire rolling machine, which is favored by the construction unit for its simple processing technology and less artificial consumption. But its thread appearance quality is poor, thread shape is incomplete, thread taper is big, connection quality is unstable, silk head burr is too much and very easy to rust, connection is extremely difficult, its use is often criticized, engineering use is also less.

\subsection{Ribbed rolling straight thread connection}

The end surface of the steel bar is first cut to strip the rib, then the irregular surface with rib is turned into a circle, and then the thread is rolled and processed. The ribbing and rolling of rebar are finished in the same equipment at the same time, the equipment is simple, the operator is few, the appearance quality of rebar thread is good-looking, this kind of technology not only in bridge engineering, but also in nuclear power, building construction and other fields, has been widely used at present. However, due to the ribbed cutting, the damage of the area of reinforcement is caused, which is easy to be destroyed first in the threaded part of reinforcement outside the sleeve, resulting in the connection quality is often difficult to meet the requirements of class I joints and other problems. The reason is that the machining quality of sleeve and silk head is not qualified and the field connection requirements are not up to standard. In particular, this process is not suitable for the steel cage part, because the threaded connection used in the design and development of the steel cage is a thick straight thread rather than a straight ribbed rolling thread, the ribbed rolling extension wire heel can not meet the requirements of class I joint, spot inspection is also not qualified. Even in the process of building construction, the qualified rate of sampling inspection of ribbed rolling tie joints from quality inspection department is only $30 \%$.

The above three straight thread connections are widely welcomed by construction units due to their features of prefabrication, no equipment required for connection, single connection (the steel can be rotated when connecting the steel), quick and convenient connection and so on. However, in the connection of steel cage of bridge foundation with straight thread, it is not possible to realize $100 \%$ reliable connection of steel bars.

\section{The main problems of straight thread reinforcement in bridge}

Bridge steel cage connection is the most difficult and problematic mechanical connection in all engineering fields.

\subsection{Technical Difficulties of steel cage connection}

3.1.1 Local adjustment is difficult. Reinforcement cage is a kind of "reinforcement bar", which is made up of many rebar (dozens or even hundreds of rebar) prefabricated into a cage on the ground, often in a vertical state with another rebar cage one by one. When connected, the connected steel bar is fixed and cannot be rotated, and the steel bar cannot move in a series along the axis.

3.1.2 Time is tight. Many reinforcement cages of bridge foundation are located below the water surface. Considering that "pit collapse" will occur after excavation of the reinforcement cage after a long time, the quality of the reinforcement cage will be affected, so it is hoped that the connection can be completed quickly when the reinforcement cage is docking.

3.1.3 High positioning accuracy. For the use of mechanical joint connection, it is also required that the position of the steel bar and the steel bar butt, to be connected to the large axial gap or offset will affect the quality and efficiency of the connection. It is difficult to take effective remedial measures in case of unconnectable rebar on site. There are many Bridges in the lower tower of the steel cage depth of tens or even hundreds of meters, in order to ensure the accuracy of the butt position, many of the use of a line on the ground, section by section of the steel cage connected in advance on the ground and do a good countersign, and then open section by section installation. This approach requires a lot of space on site, which many projects cannot do. And, even then, there will still be a lot of rebar that won't connect because of other problems.

\subsection{The main problem of connecting bridge reinforcement cage with straight thread reinforcement}

One end of the connecting rebar is processed into an extended thread (usually upper rebar, with the length of the rebar thread no shorter than the full length of the connecting sleeve), and one end is a standard thread (half of the length of the connecting sleeve). The flaring section of the connecting sleeve is to ensure that the rebar is not aligned in a convenient way. When connecting, first screw the sleeve to one end of the long thread, with the flaking 
section facing down not exceeding the exposed wire head of the reinforcement.

In order to maintain the position of the lower part of reinforcing cage, often uses the support bar crossed reinforcing cage and bear in reinforcing cage horizontal reinforcement and excavation pit location, upper reinforced falls to the lower surface on reinforcing cage after stay in this position, the crane needs to be hanging upside of reinforcing cage, until all the steel bar connection is complete, crane after the demolition of the pit support bar and the upper reinforcement cage was in pithead fixed position, connect again in the next section reinforcing cage. In this connection process, when the ribbed rolling straight thread connection is adopted, the joint quality problems easily occur:

- Due to the limitation of the site, it is impossible to pre-assemble dozens of meters of steel cage on the ground on the site, resulting in excessive position error of the connecting steel bars and time-consuming and laborious connection of the steel cage. When the offset is large, even if the connecting reinforcement is corrected, the steel wire head cannot be completely screwed into the connecting sleeve due to the excessive tilt Angle of the reinforcement.

- The "mouth" expanding joint connection, long thread end due to stripping rib technique, has been weakened by the steel section, after the joint connection tend to be at the side of the exposed steel wire head before reinforcement, the strength of the joint is difficult to guarantee the strength requirement of grade I joint (the joint of on-site inspection standard is used mostly in advance directly processed into "standard" straight thread joint inspection, rather than a true connection using "flaring" extended thread joint), joint inspection does not accord with the real state of the use of joint completely.
- In order to further ensure the quality of reinforced mechanical connection, the new industry standard "regulations of technology of reinforced mechanical connection" JTG107-2016 has clear requirements in the "all kinds of types and type of joint should be process inspection, inspection items including the single tensile strength and residual deformation limit", therefore, to solve the joint deformation performance requirements must be in the ends of each "mouth" expanding joint add a lock nut to ensure its deformation performance requirements, this will increase the connection of the workload, reduce connection efficiency, increase the cost of connection, In addition, the quality defects of the exposed filament head in the ribbed rolling process which is difficult to meet the strength requirements of class I joints will be completely exposed.

- Due to the dislocation of the axial position of the upper and lower steel wire heads of the connecting steel bar (more than $0.2-0.3 \mathrm{~mm}$ ), the upper and lower threads are not in the same spiral path line, and the threaded connection sleeve cannot screw into the lower steel bar threads, which is also a major reason for the failure of the continuous connection of the steel bar. At the same time, when the steel cage is assembled on the ground and connected to the steel cage when the position is not completely consistent, axial dislocation will occur, and the larger the diameter of the steel cage is, the more likely the above situation will occur.

- The threading gap between the end of the long thread of steel in the connecting sleeve and the threading of the connecting sleeve is too large, and the connecting strength is low, resulting in the failure of "slipping off" when the steel wire head is pulled out from the connecting sleeve.

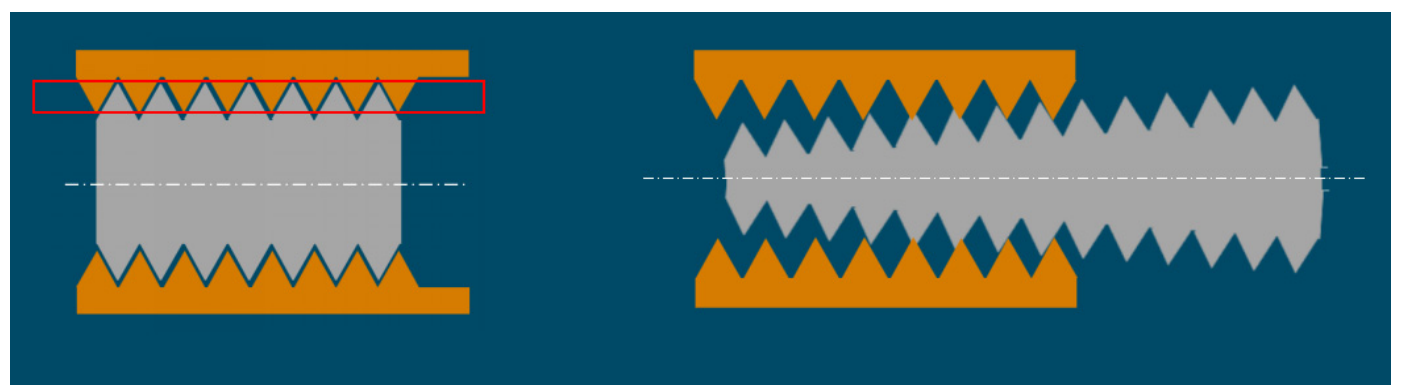

Fig 2. The End Cone of Long Thread of Reinforcement is too Large

According to incomplete statistics, $100 \%$ due to the above reinforcing cage can't realize the connection between steel individual items for more than $10 \%$, individual workers in order to cover up the fact that, while construction quality inspection personnel can't see at night, no matter how many will be reinforced can connect down pouring concrete, and this cause serious damage to the engineering quality and safety.

\section{Technical scheme for connecting the steel cage with taper sleeve locking steel joint}

According to the above analysis of the technical difficulties and problems of connecting steel cage of bridge with straight thread, in order to improve the reliability of connecting steel cage of bridge, the technical scheme of connecting steel cage of bridge with taper sleeve locking steel joint is put forward, which can 
effectively improve the reliability of connecting steel cage.

\subsection{Structure, principle and characteristics of taper sleeve locking steel joint}

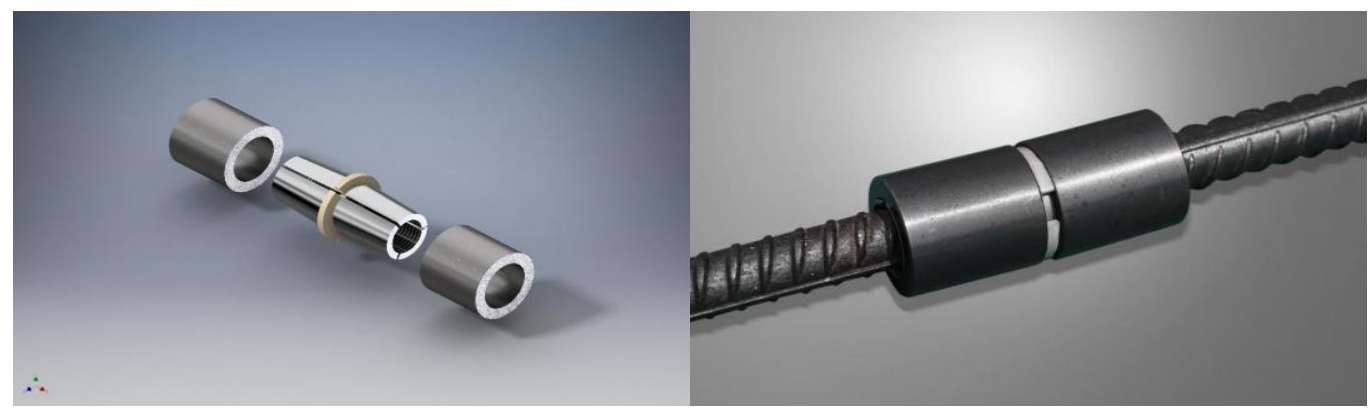

Fig 3. Structural Drawing of Locking Joint of Cone Sleeve

4.1.2 Connection principle: The outside arc surface of cone, inner surface with tooth chip lock plate will be connected the two root surface without any processing wrapped in the ribbed steel bar, use special tools along the axial direction inward extruding on the lateral 2 taper
4.1.1 Taper sleeve locking steel joint structure. The cone-Sleeve locking reinforcement joint has a special structure. Its connecting assembly is composed of two cone-sleeves, a pair of locking plates and a locking plate holder, as shown in Figure 3.
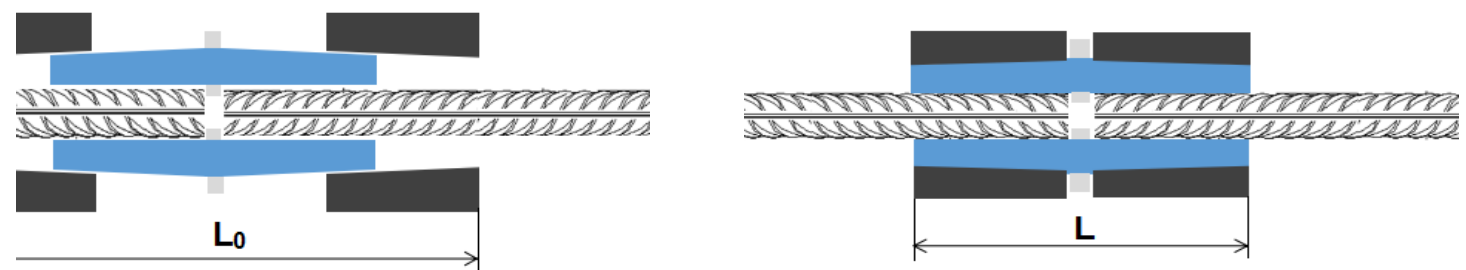

Fig 4. Before and after Connection of Taper Sleeve Locking Joint

4.1.3 Cone-Sleeve joint connection features: The reinforcement does not need to be threaded in advance, saving professional reinforcement processing personnel, equipment and site; The connection can be completed at one time on the construction work surface; The steel bar does not need to rotate when connecting, so it is easy to operate. It only takes 20-30 seconds to connect a joint, which is easy and fast to operate. The connection quality is reliable, the performance is stable, can $100 \%$ meet the national industry standard JGJ107 in class I 500MPa connector performance requirements; The position degree of the connecting bar is not required high, especially suitable for the mechanical connection of multiple bars and steel mesh. It is especially suitable for connecting the steel bar ends that cannot be machined and the steel bar joint specimens can be intercepted in engineering.

\subsection{Characteristics of connecting bridge reinforcement cage with taper sleeve locking reinforcement joint}

Taper sleeve locking reinforcement joint technology has the following characteristics when connecting reinforcement cage: sleeve, lock the lock plate along the radial compression bar, lock piece of inner gear with steel mesh, steel to stay connected, lock plate and the taper sleeve bite tight and close formation as a whole, so as to realize the connection of steel. Figure 4 shows what happens before and after the connection.
- Design the reinforcing steel parts according to the specific conditions of the project and the crane capacity;

- Prefabrication of special molding tools;

- Use special spreader for reinforcing steel parts;

- Design special tooling and tools that can solve the problem of rapid placement of reinforcement parts and reduce the auxiliary connecting time;

- Job division, organization and training for operators.

\subsection{Technical scheme of connecting bridge reinforcement cage with taper sleeve locking reinforcement joint}

\subsubsection{Technological process of taper sleeve joint connection}

- Cut the material at the end of the connecting steel bar (no horseshoe shape) and make the connecting mark line;

- Pre-install the cone sleeve according to the connection direction of the cone sleeve before the rebar to be connected (parts) is in place; 
- The connecting rebar shall be in place. For the standard type joint, the connecting gap of the connecting rebar shall be no more than $20 \mathrm{~mm}$;

- Install the lock piece and the cone sleeve: install the lock piece from the connection side and align it in the center, then clamp both sides of the cone sleeve to the middle;

- Use special squeeze pliers for taper sleeve locking joint to press both sides of the joint into the middle;

\subsubsection{Site appearance inspection of joint}

- After the completion of the connection, special caliper gage is used to check the outer connection length $\mathrm{L}$ value of the extruded cone sleeve when it conforms to the size required by the operation rules, and the connection is qualified. See Figure5. length measurement diagram of taper sleeve locking steel joint after connection.

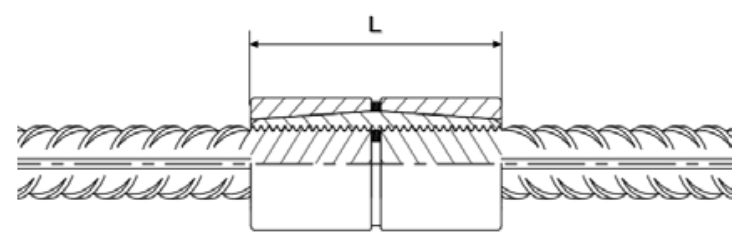

Fig 5. Length Measurement of Tapered Sleeve Locking Steel Bar Joint after Connection

- When the reinforcement is misaligned, it can be connected. See the figure 6 , which is a schematic diagram of the position error of the reinforcement that can be connected by the locking joint of cone sleeve.

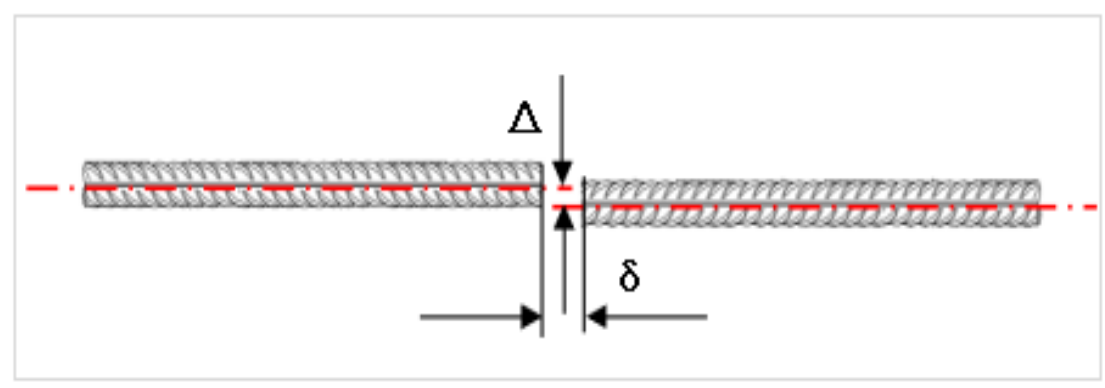

Fig 6. Schematic Diagram of Position Error of Reinforcing Bar for Connection of Taper Sleeve Locking Joint

\subsection{Taper sleeve locking reinforcement joint technology can improve the reliability of bridge reinforcement cage connection}

\subsubsection{Ensure the original strength of reinforcement}

There is no need to carry out the ribbing process, so that the steel section is weakened, avoid the exposed side of the steel wire after the joint connection before the reinforcement, to ensure the strength of the joint to meet the strength requirements of class I joint.

\subsubsection{Ensure that the reinforcement in the cone sleeve does not trip}

There will be no excessive gap between the end of the steel bar in the connecting sleeve and the cone sleeve, which will reduce the connection strength. Moreover, there will be no "slip" failure when the steel bar head is pulled out of the connecting sleeve.

\subsubsection{Ensure the smooth connection of reinforcement}

When the axial positions of the upper and lower steel wire heads of the connecting steel bar are staggered (over 0.2$0.3 \mathrm{~mm}$ ), the upper and lower threads are not on the same spiral trajectory line, and the reinforcement is basically connected smoothly by adjusting the Angle of cone sleeve. In particular, when the steel cage is assembled on the ground and connected with the steel cage hoisting position is not exactly the same and the resulting axial dislocation occurs, the conical cylinder can be used to carry out the smooth connection. See Figure 9. Rebar correction diagram of site connecting steel position.

\subsubsection{The steel cage parts are installed conveniently on site}

There is no need to screw the steel bar into the connecting sleeve during installation, which solves the problem that the position error of the connecting steel bar is too large to be installed on site after the hoisting of the steel cage part. It is reflected in the flexible local adjustment, loose positioning precision and short connecting time in the process of reinforcing steel parts butt joint.

\section{Conclusion}

In October 2016, the cone sleeve locking steel joint and steel mesh sheet technology were first applied to the main tower on the east bank of Nizhou Shuilou Bridge of Humen No.2 Bridge (Section S4). Mesh sheet technology was adopted in the 29th concrete pouring section, and the construction of connection and binding of $40 \mathrm{~mm}$ steel bars on the outer layer was shortened from the original 20 hours to 6 hours. It is expected that in the future construction of bridge reinforcement cage and main tower, under the condition of crane matching, the "overall scheme of reinforcement parts" can be completely adopted, which can further improve the construction efficiency, reduce the 
workload of the construction surface, and improve the construction efficiency of reinforcement project by about $20 \%$.

\section{References}

1. Su, Q.K., Xie, H.B. (2016) Summary of Steel Structure Bridge Construction of Hong Kong Zhuhai Macao Bridge. Chinese Journal of Highway., 29:1-9.

2. Jiang, H.T. (2020) Safety Assessment Method for the Influence of High-Speed Railway Bridge Construction on Power Station Operation. Journal of Chongqing Jiaotong University (Natural Science Edition)., 39 : 117-122.

3. Zhao, G.W. (2015) Research on Bridge Life Prediction based on Reliability Theory. Highway., 60:122-125.

4. Liang, W., Zhuo, W.D. (2014) Bridge Life Cycle Cost Analysis Method Considering Environmental Impact. Journal of Guangxi University(Natural Science Edition)., 39:854-863.

5. Zhou, D.C., Liang, Z.C., Shao J.H. (2014) Research on Displacement Ductility of Reinforced Concrete Pier Column of Highway Bridge. Earthquake Engineering and Engineering Vibration., 34:62-67.

6. Li, J., Chen, H., Sun L.F. (2013) Carbonation Durability Prediction of Reinforced Concrete Members of a Bridge under Construction. Journal of Zhengzhou University (Science Edition), 45:110-114.

7. Zhou, H.F., Gong, J.X., Zhang, Y.L. (2012) Research on Extramural Reliability of Reinforced Concrete Members of Railway Bridges. Journal of Railway Science and Engineering., 4:8-13. 